\title{
In vivo Interictal Neuronal Signatures of Human Periventricular Nodular
}

\section{Heterotopia}

Valerio Frazzini, MD, $\mathrm{PhD}^{1,2,3_{*}}$ Stephen Whitmarsh, $\mathrm{PhD},{ }^{2}{ }^{2}$ Katia Lehongre, $\mathrm{PhD},{ }^{2}$ Pierre Yger, $\mathrm{PhD},{ }^{4}$ Jean-Didier Lemarechal, $\mathrm{PhD},{ }^{2}$ Bertrand Mathon, MD,${ }^{2,3,5}$ Claude Adam, MD, PhD, ${ }^{1}$ Dominique Hasboun, MD, PhD, ${ }^{2,3,6}$ Virginie Lambrecq, MD, PhD, ${ }^{1,2,3}$ and Vincent Navarro, $\mathrm{MD}, \mathrm{PhD},{ }^{1,2,3 \dagger}$

1 AP-HP, Pitié Salpêtrière Hospital, Epilepsy Unit and Reference Center for Rare Epilepsies, F75013, Paris, France

2 Paris Brain Institute, ICM, INSERM, CNRS, F-75013, Paris, France

3 Sorbonne Université, F-75013, Paris France

4 Institut de la Vision, INSERM UMRS 968, UPMC UM 80, France

5 AP-HP, Pitié Salpêtrière Hospital, Department of Neurosurgery, F-75013, Paris, France

6 AP-HP, Pitié Salpêtrière Hospital, Department de Neuroradiology, F-75013, Paris, France

* Contributed equally to this study.

Corresponding author: Pr Vincent Navarro 
Corresponding author's address: Epilepsy Unit, Pitié-Salpêtrière Hospital, 47 boulevard de l’Hôpital, 75651 Paris cedex 13, France

Corresponding author's phone and fax: Tel: 331421619 40, Fax: 33142161942

Corresponding author’s e-mail address: vincent.navarro@aphp.fr

ORCID: Valerio Frazzini 0000-0003-1187-3352, Stephen Whitmarsh 0000-0002-3969-6565, Pierre Yger 0000-0003-1376-5240, Vincent Navarro 0000-0003-0077-8114 


\begin{abstract}
Periventricular nodular heterotopia $(\mathrm{PNH})$ is a common type of malformation of cortical development and a cause of drug-resistant epilepsy. In contrast with other cortical malformations, no consistent interictal patterns have yet been identified in $\mathrm{PNH}$, and it remains controversial whether epileptic activity originates within nodules. The current study addresses the heterogeneity in LFP signatures, as well as the question of epileptogenicity of nodules, by means of microelectrode recordings implanted within PNH tissues in two epileptic patients. Microelectrodes also allowed the first in vivo recording of heterotopic neurons in humans, permitting the investigation of neuronal firing rates during patterns of interictal activity. Highly consistent interictal patterns (IPs) were identified within PNH: 1) trains of periodic slow waves and 2) isolated slow deflections, both with superimposed fast activity, and 3) epileptic spikes. Neuron firing rates were significantly modulated during all IPs, suggesting that different IPs were generated by the same local neuronal populations. To conclude, this study presents the first in vivo microscopic description of local $\mathrm{PNH}$ microcircuits in humans and their organization into multiple epileptic neurophysiological patterns, providing a first pathognomonic signature of human PNH.
\end{abstract}




\section{Introduction}

Malformations of cortical development (MCD) are frequently associated with epileptic seizures. The underlying epileptogenic process can sometimes be inferred from patterns of interictal activity that are known to correlate with specific epileptogenic lesions. For example, patients with type-IIb focal cortical dysplasia often show localized periodic spikes or poly-spikes (Palmini et al., 1995) and persistent rhythmic fast-frequency discharges of very high amplitude (>100 $\mu \mathrm{V}$ ) are typical of lissencephaly (Gastaut et al., 1987). In periventricular nodular heterotopia $(\mathrm{PNH})$, reports of interictal patterns have not yet converged onto stereotypical patterns that could help its identification. PNH is one of the most common types of MCD (Guerrini and Dobyns, 2014), resulting from an abnormal neuronal migration process wherein clusters of neurons form nodular masses of gray matter close to the walls of the lateral ventricles (Dubeau et al., 1995). The majority of patients with PNH suffer from focal pharmaco-resistant epilepsy (Battaglia et al., 2006). One sEEG study found an absence of EEG background activity in $\mathrm{PNH}$, together with low voltage, low frequency activity, frequent high voltage spikes and positive waves (Tassi et al., 2004), while another study only reported frequent low voltage spikes-and-waves (Mirandola et al., 2017). Ripples (80-250 Hz) and fast ripples (250-500 Hz) have also been recorded (Jacobs et al., 2009; Ferrari-Marinho et al., 2015). The heterogeneity in sEEG findings might be caused partly by the fact that neurons within the ectopic tissue are highly disorganized (Dubeau et al., 1995). This would limits spatial summation of electric currents measured by regular sEEG electrodes. Furthermore, it would make local field potentials (LFPs) highly susceptible from spread of activity originating in surrounding tissue. Implanted microelectrodes, however, due to their high impedance, only record LFPs and action potentials from nearby $(\approx 140 \mu \mathrm{m})$ neurons, and are less dependent on the spatial summation of current flow 
(Buzsaki, 2004). This spatial selectivity allows the unequivocal measurement of ectopic neurons.

The current study therefor attempts to address the heterogeneity in LFP signatures of PNH by means of microelectrode recordings within PNH tissue.

In Type II focal cortical dysplasia, epileptic discharges are generated autonomously within the lesion (Avoli et al., 1999; Najm et al., 2007). In sub-cortical MCD, however, the origins of epileptic discharges are less clear-cut and might lie both within the MCD and adjacent cortical structures. For example, in a preclinical model of subcortical band heterotopia, interictal activities were localized in the normal overlying cortex, with heterotopic lesions only secondarily involved in the epileptic process (Petit et al., 2016). In PNH, seizure onset zones (SOZ) have been localized either within the lesion, within the adjacent cortex, or in both (Kothare et al., 1998; Tassi et al., 2004; Battaglia et al., 2006; Valton et al., 2008; Mirandola et al., 2017; Pizzo et al., 2017). While the question whether nodules are typically part of the SOZ in PNH is beyond the scope of the current paper, the relationship between interictal signatures of PNH and the SOZ will be addressed. Specifically, we will evaluate whether interictal patterns are associated with the SOZ, as determined by clinical evaluation.

On the microscopic level, very little is known about the function of individual heterotopic neurons. In a methylazoxymethanol model, rat heterotopic neurons were found to be more excitable as result of a deficient A-type potassium current (Castro, et al., 2001), while NR2A and NR2B subunit expression and regulatory molecular machinery have been found to be reduced in preclinical rat models (Colacitti et al., 1999) as well as in resected human PNH tissue (Finardi et al., 2006). Moreover, a previous in vitro study found a dramatic increase in inhibitory GABAergic synaptic transmission of heterotopic neurons (Kitaura et al., 2012), which could reflect a compensating drive against the otherwise hyper-excitability of the pathological network 
of PNH (Calcagnotto, et al., 2002). To the best of our knowledge, no study has yet reported in vivo behavior of heterotopic neurons in humans. Based on the above, we expect a pathological increase in excitability of heterotopic neurons within $\mathrm{PNH}$, expressed as increased and highly correlated activity during interictal LFP patterns.

The goal of this study was to i) describe unique and consistent LFP signatures in PNH nodules, and ii) to describe the concomitant neuronal behavior of the heterotopic neurons. For this purpose, interictal patterns (IPs) were described and validated at an unprecedented level of microscopic detail by means of microelectrode recordings from three PNH nodules in two patients. LFP patterns were identified on the basis of their morphological and pathological features and described in the time and frequency domains. Single units were extracted using spike-sorting algorithms and their firing rate time-locked and correlated with LFP of the IPs. In an exploratory analysis, spike shapes were used to classify neurons into putative pyramidal and interneurons. We expected to find i) consistent IPs within and between all three nodules, and ii) hyper excitable neuronal firing patterns associated with the IPs.

\section{Materials and methods}

\section{sEEG recording}

All intracranial sEEG procedures were performed according to clinical practice and the epilepsy features of the patients (see patient information in supplemental materials for clinical details). Two (Patient 1) and three (Patient 2) Behnke-Fried type macro-micro electrodes (AdTech®) were inserted (Fried et al., 1999). In patient 1, one macro-micro electrode targeted one nodule in the left hemisphere. In patient 2, one macro-micro electrode targeted one nodule in the left 
hemisphere, and another one targeted a second nodule in the right hemisphere (see supplementary Table 1). Signals from macro- and microelectrodes were continuously and synchronously recorded at $4 \mathrm{kHz}$ and $32 \mathrm{kHz}$, respectively, using a hardware filter at $0.01 \mathrm{~Hz}$ (Atlas Recording System; NeuraLynx, Tucson, AZ, USA). Post-implantation electrode locations were based on a pre-implantation 3T 3D-MRI, post-implant 1.5T 3D-MRI and CT scan, integrated using the Epiloc toolbox (Pérez-Garcia et al., 2015), an in-house developed plugin for the 3D-Slicer visualization software (Fedorov et al., 2012). Patients gave a written informed consent (project C11-16 conducted by INSERM and approved by the local ethic committee, CPP Paris VI).

\section{Visual analysis of micro-LFP patterns}

Pathological activities were visually identified on microelectrodes signals, according to traditional morphological characteristics used in clinical practice, then classified into different interictal patterns (IPs). Criteria for each pattern were then used for a complete manual scoring of continuous data recorded at the first day after implantation. Data from Patient 1 were analyzed for 24 hours. Data from Patient 2, due to richness of interictal activity, were analyzed for 6 hours. For each nodule, the annotation of the IPs was based on the LFP from a single selected microwire, using a software developed in-house (MUSE). Onset and duration of slow waves were determined manually on a subset of annotated patterns. Periodicity was determined in accordance with Hirsch et al. (2013), by calculating the percentage of slow waves that deviated less than $25 \%$ from the average period within each pattern. Only sequences with at least 3 consecutive slow waves were considered. 


\section{Seizure onset zone}

SOZ was determined as part of the standard clinical procedure, involving visual annotation and analyses of the entire recording by trained epileptologists (CA, VF \& VN). Because both patients were not selected for surgical intervention, post-surgical validation of SOZ was not available.

\section{Spectral analysis of micro-LFP patterns}

Analyses were performed with a combination of FieldTrip (Oostenveld, et al., 2011) and custom MATLAB scripts (The Mathworks Inc., Natick, Massachusetts). Time-courses were first aligned according to the max/min peak of filtered data (Supplementary Table 2). Data were then downsampled to $1 \mathrm{kHz}$. Average LFPs were based on the aligned time-courses. Time-frequency representations (TFRs) were calculated using a Hanning taper applied to an adaptive sliding time window of seven cycles $(t=7 / f, \Delta t=10 \mathrm{~ms})$. Average power in $10 \mathrm{~Hz}-200 \mathrm{~Hz}$ was expressed in percentage change, relative to the baseline-period at $-2 \mathrm{~s}$ to $-1 \mathrm{~s}$ relative to pattern onset. For epileptic spikes, a baseline period of $-1 \mathrm{~s}$ to $-0.5 \mathrm{~s}$ was used, except for the second nodule of Patient 2, where a baseline-period of $-2 \mathrm{~s}$ to $-1 \mathrm{~s}$ was used due to more extended fluctuations of firing rate preceding the epileptic spike.

\section{Spike sorting and analysis}

All spikes occurring during artefacted periods were ignored. After selecting electrodes that showed multi-unit activity (MUA), data were temporally whitened, and spikes were automatically detected at 6 median absolute deviations of high-pass filtered $(>500 \mathrm{~Hz})$ data. A combination of density-based clustering and template matching algorithms were used to automatically cluster the detected spikes (Yger et al., 2018). Clusters were evaluated as to 
whether they reflected putative single-unit activity (SUA) or multi-unit activity, based on the inter-spike-interval (ISI), the percentage of refractory period violation (RPV=ISI $<2 \mathrm{~ms}$ ) and spike morphology. Resting behavior of detected units were further explored by mean firing rate and Coefficient of Variation (Shinomoto, et al., 2003) calculated on the baseline period preceding epileptic spikes to maximize data of consistent resting behavior. Spike-times were epoched and time-locked identically to the LFP analyses. Average spike rates were calculated continuously at $1000 \mathrm{~Hz}$, using a Gaussian smoothing kernel of 12.5ms for Epileptic Spikes, and 50ms for the longer patterns to better capture the slower modulations of firing rate. Correlations were calculated between average time-locked spike-rates of each unit, and the average time-locked LFP of every pattern. Finally, spike-rates of each trial were binned into 200 bins for statistical analyses. To control for multiple comparisons and non-normal distributions of firing-rates, we performed non-parametric cluster-based permutation tests (Maris \& Oostenveld, 2007) to determine time-periods where firing-rates changed significantly from baseline. A threshold of $p<0.01$ (first-level t-test) was used to determine contiguous clusters, after which a threshold of $p<0.05$ (one-sided correction) determined whether the clusters (sum of t-values) could be explained under permutation $(n=1000)$. Finally, an exploratory classification of detected units into putative pyramidal and interneurons was based on the half-width duration and trough-peak time of their waveforms (c.f. Bartho et al., 2004).

\section{Results}

\section{Epileptic LFP activities revealed by microelectrodes in PNH}

Three IPs were consistently detected by microelectrodes in the nodules (Fig. 1 \& Fig. 2; See Supplementary Fig.1 for an example of three patterns within a single time window). Epoch-by- 
epoch correlations between the microelectrode and adjacent macroelectrode contacts, showed that, besides the microelectrodes, all IPs were only recorded in the most profound macro contacts, located within the PNH (Fig. 2 E). The patterns occurred very frequently, often within seconds of each other. The mode $(\lambda)$ of each pattern (i.e. the most frequent time interval between events) will be reported (see supplementary Fig. 2 for distributions).

[Insert Figure 1 and Figure 2 here]

\section{Periodic Slow Waves}

Periodic Slow Waves (PSW) were identified in both Nodule 1 (Fig. 1C right panel, n=1882, $\lambda=2.48 \mathrm{~s}$ ) and Nodule 2 (Fig. $1 \mathrm{~F}$ right panel, $\mathrm{n}=973, \lambda=2.23 \mathrm{~s}$ ). These patterns were detectable on microelectrodes, and to a smaller degree on the adjacent macroelectrode contact (mainly in the nodule from patient 1, Fig. 1C, left panel: M1-M2, M2-M3). The periodicity of the PSW was clear in the average LFP of both Nodule 1 (Fig. 2A, right panel) and Nodule 2 (Fig. 2B, right panel). Average $( \pm \sigma)$ duration of a single slow wave was similar between nodules (Nodule 1: $283 \pm 74 \mathrm{~ms}$, Nodule 2: $271 \pm 72 \mathrm{~ms}$ in Nodule 2), as well as the time periods between the onset of two slow waves (Nodule 1: subset $n=181, \mathrm{t}=511 \pm 148 \mathrm{~ms}$, Nodule 2: subset $n=153, \mathrm{t}=574 \pm 152 \mathrm{~ms}$. Of these time periods, $83 \%$ and $79 \%$ varied less than $25 \%$ from the average period, respectively, in accordance with periodicity. Low voltage fast activity (FA) was superimposed on the slow waves in both Nodules with a mean peak at $112 \mathrm{~Hz}$ and $137 \mathrm{~Hz}$, respectively, and was modulated in accordance with the periodicity of the slow waves. (Fig. 1C\&F).

\section{Isolated trains of Fast Activity}

Isolated trains of FA were identified on microelectrodes located in both Nodule 1 ( $\mathrm{n}=1005$, $\lambda=1.89 \mathrm{~s})$ and Nodule $2(\mathrm{n}=93, \lambda=8.19 \mathrm{~s})$. In Nodule 1 , FA was usually superimposed on a 
polyphasic slow deflection (0.5s-1s, Fig. 1C, middle panel), while in Nodule 2, FA occurred at the onset of a sharply appearing slow deflection (2s-4s, Fig. 1F, middle panel). Time-frequency analysis showed that in Nodule 1 , power increased to $43 \mathrm{~Hz}$ and $96 \mathrm{~Hz}$ for $\approx 0.5 \mathrm{~s}$ during the FA (Fig. 2A, middle panel). Nodule 2 showed a narrow-band increase of power at $112 \mathrm{~Hz}$ for at least 1s (Fig. 2B, middle panel). These events were isolated, with no tendency to ictal organization. In Nodule 3, low amplitude deflections coincided with periodic short trains of FA $(n=533)$, that lasted for $326 \pm 79 \mathrm{~ms}$. Periods were $1075 \pm 241 \mathrm{~ms}$ (Fig. 1F), of which $91 \%$ varied less than $25 \%$ (subset $n=155$ ) in accordance with periodicity. Time-frequency analysis showed periodic modulation of high frequency power, centered on $110 \mathrm{~Hz}$ (Fig. 2D, right panel). FA were also detectable despite smaller amplitudes, on the macroelectrodes located in the nodules (Fig. $1 \mathrm{C}, \mathrm{F}$, I, middle panels: M1-M2, M2-M3).

\section{Epileptic Spikes}

Epileptic Spikes (ES) were identified in all three nodules on both the micro- and adjacent macroelectrodes contacts (Fig. 1). Nodule $1(n=3878, \lambda=0.59 \mathrm{~s})$ showed isolated sharp monophasic waves (Fig. 1C, left panel). In Nodule $2(n=2705, \lambda=0.50 \mathrm{~s})$, spikes were generally followed, and often preceded, by a slow wave (Fig. 1F, left panel), which was also apparent in the average time-locked LFP (Fig. 2B, left). In Nodule $3(n=403, \lambda=2.97 \mathrm{~s})$, spikes were characterized by a low amplitude di- or triphasic wave (Fig. 1I, left). Low voltage FA was superimposed on ES, with a mean peak at $113 \mathrm{~Hz}, 137 \mathrm{~Hz}$ and $126 \mathrm{~Hz}$, respectively (Fig. 2A-C, left panel).

\section{Seizure onset zones}

In patient 1 , seizures were characterized by the appearance of a slow deflection overlapped with fast rhythms between $115 \mathrm{~Hz}$ and $160 \mathrm{~Hz}$. Seizures started from the deep contacts of the two 
macroelectrodes (Fig. 1J), located in the posterior part of the PNH (Supplementary Table 1), strongly implicating the $\mathrm{PNH}$ as the SOZ. In patient 2, seizures were characterized by fast rhythms (above the $270 \mathrm{~Hz}$ ), starting in contacts located within the anterior part of the left nodule (Fig. 1K), with a very fast involvement of the posterior part of homolateral PNH and the adjacent temporal cortex. The SOZ was thus represented by a complex network involving both the left PNH and the adjacent cortex. Finally, the right nodule (in Patient 2) was not part of the SOZ but did eventually take part in later stages of seizure development.

\section{All neurons are recruited by interictal patterns}

In total, twenty-one microwires, located in $\mathrm{PNH}$, were included in the analyses (Nodule 1: $n=6$, Nodule 2: $n=7$, Nodule 3: $n=8$ ). Spike clustering resulted in 25 units (Table 1, Supplemental Fig 3) of which $8(32 \%)$ were classified as single units. Single units were shown to significantly modulate their firing rates in response to all IPs. Firing-rate increased up to $505 \%$ for PSW, 4281\% for FA, and $3840 \%$ for ES (Fig. 3A-C, Table 1: $\uparrow$ ). Most of the units also showed brief episodes of decreased firing rate surrounding the IPs, often by $\approx 100 \%$, i.e. silence (Table 1: $\downarrow$; Fig. 2 and Fig. 3A-C). Especially during ES, firing rates strongly decreased in all units within $\approx$ 500ms surrounding the discharge. Furthermore, trial-by-trial correlations showed that the modulation of firing rates was highly consistent with the timing and shape of the interictal LFP in all units (Table 1; see also: Fig. 2, Fig. 3A-C, Supplementary Fig 3). A massive increase of firing rate was shown for the very short duration of the ES, while FA was associated with a more prolonged increase of firing rate. During PSW, firing rates showed regular increases and decreases. Interestingly, three clusters, including 2 single units, significantly modulated their 
firing-rate inversely with respect to the IPs: they decreased their firing rate during interictal LFP activities (Fig. 2C: middle panel; Table 1: Nodule 2, Unit 4\&5, and Nodule 3, Unit 2).

\section{Exploratory classification of neuronal types}

An exploratory classification of single units into neuronal types was based on the half-width and trough-to-peak time of the action-potential (Fig. 3D). Values separating putative interneurons from pyramidal cells were consistent with previous reports (Trough-to-peak: $\approx 0.5 \mathrm{~ms}$, Halfamplitude duration: $\approx 0.25 \mathrm{~ms}$; Bartho et al., 2004). Seven out of 8 units were classified as putative pyramidal cells, while one was consistent with a narrow-spiking putative interneuron (Fig. 3D, action potential shapes, interneuron is identified by a star). No clear differences were observed in firing behavior although the interneuron did show the highest Fano Factor (Fig. 3D, right panel).

[Insert Figure 3 here]

\section{Discussion}

We identified two interictal patterns (IPs) in PNH that occurred frequently and with a unique and consistent morphology: Periodic Slow Waves (PSW) and isolated Slow Waves, both supporting superimposed Fast Activity (FA). In addition, Epileptic Spikes (i.e. interictal epileptic discharges, or IEDs) were shown to occur often and consistently in all three nodules. The IPs were shown to originate in the PNH and not in the adjacent cortex. Firstly, the microelectrodes were validated by imaging to be located within the PNH. The brain volume explored by the microelectrodes is extremely small due to its microscopic size and high impedance (Buzsaki, 
2004). Furthermore, the IPs correlated only with neighboring macro contacts that were also located within the PNH (Fig. 2E), providing further evidence for the PNH origin of the IPs.

The role of PNH in the initiation of seizures is still a matter of debate. PNH might be best considered to be a part of a complex pathological network that engages both nodules and cortical structures, resulting in seizures that might start in $\mathrm{PNH}$, in the overlying cortex, or in both (Kothare et al., 1998; Tassi et al., 2004; Battaglia et al., 2006; Valton et al., 2008; Mirandola et al., 2017, Pizzo et al., 2017). Interestingly, in our study, the IPs occurred within all the 3 nodules supporting the idea that while the IPs are highly localized to the PNH, their epileptogenicity is the result of their position within a larger pathological network.

On the microscopic level, we reported the striking finding that all spontaneously active neurons modulated their firing rate in accordance with the LFPs of all IPs. Such hyper-excitable state of the neuronal populations is in line with the hyper-synchronous and hyper-excitable neuronal populations described in rodent models where PNH was associated with aberrant cellular expression of ionic channels (Castro, et al., 2001, Calcagnotto, et al., 2002). However, previous microelectrode studies have shown strong heterogeneity in firing rates during cortical and mesial temporal interictal discharges (Le Van Quyen et al., 2008; Keller et al., 2010; Alvarado-Rojas et al., 2013), as well as during ictal discharges (Lambrecq et al., 2017). This discrepancy could be due to several reasons. First, our results suggest that neurons in PNH are hyper-excitable, making them perhaps more responsive, and therefore more similar, during interictal activity. Second, in contrast with previous studies, we restricted ourselves to a single lesion (PNH), and highly consistent interictal morphologies. 
Finally, we found tentative evidence for both putative pyramidal cells and interneurons, in accordance with histological studies showing both excitatory neurons and several types of interneurons in human PNH (Thom, et al., 2004; Meroni et al., 2009). We did not find evidence that specific neuronal types were associated with specific IPs. Rather, our findings suggest a hyper-excitable network in which the specific network conditions might be more important in determining which IP will occur at a particular point in time. For example, periodic patterns might result from alternating inhibition and rebound excitation in the same neuronal network (Tóth et al., 2018). Different IPs could also be the result of different dynamics of rebound excitation. In our data, the increase in firing rate during FA was more sustained than in the other patterns, while decreases in firing rate preceding FA were less obvious. In contrast, ES were preceded by strong synchronous decreases in firing rate, followed by a short and rapid peaking in firing rate. Alternatively, different subtypes of interneurons might have differentially modulated the pyramidal cells, resulting in different patterns expressed by the same pyramidal cells.

To conclude, this study presents the first in vivo microscopic description of local PNH microcircuits in humans and their organization into multiple epileptic neurophysiological patterns. PSW and FA might provide a pathognomonic signature of PNH reflecting highly pathological network activity that recruits all spontaneously active neurons. Future research will determine whether these microscopic IPs are specific electrophysiological signatures of PNH or can be found in sub-cortical heterotopia or other types of cortical malformations as well. 


\section{Acknowledgements}

We would like to thanks Pierre Pouget (ICM, Pitié-Salpêtrière hospital, Paris, France) and Fiona Francis (Institut du Fer à moulin, Paris, France) for the helpful discussions and comments on the manuscript.

\section{Funding}

This study was supported by the program "Investissements d'avenir" ANR-10-IAIHU-06, and grants from the OCIRP-ICM and the Fondation de l'APHP pour la Recherche - Marie-Laure PLV Merchandising.

\section{Competing interests}

The authors report no competing interests. 


\section{Figure descriptions}

\section{Figure 1.}

Electrode implantation into the PNH nodules and interictal LFP patterns. (A, D \& G) Brain MRI showing the electrode trajectories, exploring the three analyzed nodules (A, Patient 1, Nodule 1; D \& G, Patient 2, Nodule 2 \& 3). (B, E \& H) Schematic representation of the macromicroelectrodes (M1-M4: macroelectrode contacts; $\mu 1-\mu 8$ : microelectrodes). All the geometrical features of the electrodes are expressed in millimeters. Macroelectrode recordings $(\mathrm{C}, \mathrm{F}, \mathrm{I})$ are shown in bipolar montage. In Nodule 1 (C) and 2 (F), three LFP patterns were recorded (Epileptic Spike, Fast Activity, Periodic Slow Wave). These patterns were apparent on microelectrodes (LFP $\mu 1$ and $\mu 2$ ), and to a smaller degree on macroelectrodes (M1-M2). LFP patterns were associated with MUA recorded on the microelectrodes. In Nodule 3, two patterns were identified (I) that were apparent on the micro LFP, and to a smaller degree on the macro LFP. J) In Patient 1, seizures originated from the deeper contacts of the two electrodes located into the posterior part of the PNH. Seizures were characterized by the appearance of a slow deflection overlapped with fast rhythms between $115 \mathrm{~Hz}$ and $160 \mathrm{~Hz}$, starting from the posterior part of PNH. Asterisk on time-axis denotes end of seizure, and reappearance of an interictal pattern (periodic slow waves). Clinical symptoms included dizziness, staring, loss of consciousness and motor automatisms. K) In Patient 2, seizures were characterized by a fast rhythmic activity starting in the anterior part of the nodule, then evolving into large spike-wave discharges detectable in the posterior part of the left PNH. Clinical symptoms consisted of tingling sensations of shoulders and trunk, remounting to the head, sometimes followed by forced head rotation to the right, eyelids blinking, small vocalizations and blowing. 


\section{Figure 2.}

Multi-level description of interictal patterns. A-D: Top panels show time-frequency representations (TFRs). Average power in $10 \mathrm{~Hz}-200 \mathrm{~Hz}$ was expressed in percentage change, relative to the baseline-period. Top panel overlays show time-courses aligned according to the max/min peak. Top panel inlays show average spectra. Middle panels show time-locked raster plots of spike times. Bottom panels shows corresponding time-locked firing-rates, with timeclusters of significant change (yellow=increase, blue=decrease), compared to baseline (arrow). E: Correlations between LFP of micro- and macroelectrode contacts (bipolar montage) shows the strongest correlation with macroelectrode contacts closest to microelectrodes (yellow=positive, blue=negative, marker-size $=$ absolute Pearson's $\rho$ ). Note an inversion (negative correlation) for macroelectrode contacts further away from the microelectrodes, showing that the dipolar sources of the LFPs were adjacent to the microelectrodes.

\section{Figure 3.}

Characterization of single neuron features and behaviors. Description of single unit behavior for Nodule 1 (A), Nodule 2 (B), and Nodule 3 (C), including template waveform, inter-spike-interval (ISI) histogram and firing-rates time-locked to IPs. Significant increases and decreases in firingrate compared to baseline are indicated in yellow and blue, respectively. D) Unit classification (left). Trough-to-peak plotted against half-amplitude duration, resulting in two clusters of neuronal types. Pyramidal (Pyr) cells cluster on the top right, interneurons (Int) on the bottomleft. Filled symbols indicate single-unit activity (SUA), empty circles multi-unit activity (MUA). Average waveforms and 1000 overlaid responses show putative pyramidal cell (upside-down 
pyramid shape), and putative narrow-spiking interneuron (asterisk). Putative pyramidal cells and interneurons were based on half-width duration and trough-peak time of their waveforms (c.f. Bartho et al., 2004). Firing behaviors (right) were characterized by mean firing rate (FR), Coefficient of Variation (CV), and CV2, averaged over 60 s segments of the continuous data. 


\section{References}

Alvarado-Rojas, C., Lehongre, K., Bagdasaryan, J., Bragin, A., Staba, R., Engel, J., Le Van Quyen, M., et al. (2013). Single-unit activities during epileptic discharges in the human hippocampal formation. Frontiers in Computational Neuroscience, 7, 140. doi: 10.3389 /fncom. 2013.00140

Avoli M., Bernasconi A., Mattia D., Olivier A, Hwa G-G. (1999). Epileptiform discharges in the human dysplastic neocortex: in vitro physiology and pharmacology. Ann Neurol. 1999 Dec;46(6):816-26.

Bartho, P., Hirase, H., Monconduit, L., Zugaro, M., Harris, K. D., \& Buzsaki, G. (2004). Characterization of Neocortical Principal Cells and Interneurons by Network Interactions and Extracellular Features. Journal of Neurophysiology, 92(1), 600-608. doi: 10.1152/jn.01170.2003

Battaglia, G., Chiapparini, L., Franceschetti, S., Freri, E., Tassi, L., Bassanini, S., Granata, T., et al. (2006). Periventricular Nodular Heterotopia: Classification, Epileptic History, and Genesis of Epileptic Discharges. Epilepsia, 47(1), 86-97. doi:10.1111/j.1528-1167.2006.00374 .x

Buzsaki, G. (2004). Large-scale recording of neuronal ensembles. Nature Neuroscience, 7(5), 446-451. doi: 10.1038 /nn 1233

Calcagnotto, M. E., Paredes, M. F., \& Baraban, S. C. (2002). Heterotopic neurons with altered inhibitory synaptic function in an animal model of malformation-associated epilepsy. The Journal of Neuroscience: The Official Journal of the Society for Neuroscience, 22(17), 75967605. 
Castro, P. A., Cooper, E. C., Lowenstein, D. H., \& Baraban, S. C. (2001). Hippocampal heterotopia lack functional Kv4.2 potassium channels in the methylazoxymethanol model of cortical malformations and epilepsy. The Journal of Neuroscience: The Official Journal of the Society for Neuroscience, 21(17), 6626-6634.

Colacitti, C., Sancini, G., DeBiasi, S., Franceschetti, S., Caputi, A., Frassoni, C., Battaglia, G., et al. (1999). Prenatal methylazoxymethanol treatment in rats produces brain abnormalities with morphological similarities to human developmental brain dysgeneses. Journal of Neuropathology and Experimental Neurology, 58(1), 92-106. doi: 10.1097/00005072-199901000-00010

Constantinidis, C., \& Goldman-Rakic, P. S. (2002). Correlated Discharges Among Putative Pyramidal Neurons and Interneurons in the Primate Prefrontal Cortex. Journal of Neurophysiology, 88(6), 3487-3497. doi: 10.1152/jn.00188.2002

Dubeau, F., Tampieri, D., Lee, N., Andermann, E., Carpenter, S., Leblanc, R., Andermann, F., et al. (1995). Periventricular and subcortical nodular heterotopia A study of 33 patients. Brain, 118(5), 1273-1287. doi: 10.1093 /brain/ 118.5.1273

Fedorov, A., Beichel, R., Kalpathy-Cramer, J., Finet, J., Fillion-Robin, J.-C., Pujol, S., Kikinis, R., et al. (2012). 3d Slicer as an image computing platform for the Quantitative Imaging Network. Magnetic Resonance Imaging, 30(9), 1323-1341. doi: 10.1016/j.mri. 2012.05.001

Ferrari-Marinho T., Perucca P., Mok K., Olivier A., Hall J., Dubeau F., Gotman J. (2015). Pathologic substrates of focal epilepsy influence the generation of high-frequency oscillations. Epilepsia, Apr;56(4):592-8. doi: 10.1111/epi.12940. 
Finardi, A., Gardoni, F., Bassanini, S., Lasio, G., Cossu, M., Tassi, L., Battaglia, G., et al. (2006). NMDA receptor composition differs among anatomically diverse malformations of cortical development. Journal of Neuropathology and Experimental Neurology, 65(9), 883-893. doi: 10.1097/01.jnen.0000235117.67558.6d

Fried, I., Wilson, C. L., Maidment, N. T., Engel, J., Behnke, E., Fields, T. A., Ackerson, L. et al., (1999). Cerebral microdialysis combined with single-neuron and electroencephalographic recording in neurosurgical patients. Technical note. Journal of Neurosurgery, 91(4), 697-705. doi: 10.3171/jns.1999.91.4.0697

Gastaut, H., Pinsard, N., Raybaud, C., Aicardi J., Zifkin, B. (1987) Lissencephaly (agyriapachygyria): clinical findings and serial EEG studies. Dev Med Child Neurol.1987; 29:167-180.

Guerrini, R., \& Dobyns, W. B. (2014). Malformations of cortical development: clinical features and genetic causes. The Lancet Neurology, 13(7), 710-726. doi: 10.1016/S14744422(14)70040-7

Henze, D. A., Borhegyi, Z., Csicsvari, J., Mamiya, A., Harris, K. D., Buzsaki, G. (2000). Intracellular Features Predicted by Extracellular Recordings in the Hippocampus In Vivo. Journal of Neurophysiology, 84(1), 390-400. doi: 10.1152/jn.2000.84.1.390

Hirsch, L. J., LaRoche, S. M., Gaspard, N., Gerard, E., Svoronos, A., Herman, S. T., Drislane, F. W., et al. (2013). American Clinical Neurophysiology Society’s Standardized Critical Care EEG Terminology: 2012 version. Journal of Clinical Neurophysiology, 30(1), 27.

Jacobs J., Levan P., Châtillon C.E., Olivier A., Dubeau F., Gotman J. (2009) High frequency oscillations in intracranial EEGs mark epileptogenicity rather than lesion type. Brain. Apr;132(Pt 4):1022-37. doi: 10.1093/brain/awn351. 
Keller, C. J., Truccolo, W., Gale, J. T., Eskandar, E., Thesen, T., Carlson, C., Cash, S., et al. (2010). Heterogeneous neuronal firing patterns during interictal epileptiform discharges in the human cortex. Brain, 133(6), 1668-1681. doi: 10.1093 /brain/awq 112

Kitaura, H., Oishi, M., Takei, N., Fu, Y.-J., Hiraishi, T., Fukuda, M., Kakita, A., et al. (2012). Periventricular nodular heterotopia functionally couples with the overlying hippocampus: Optical Imaging of PVNH. Epilepsia, 53(7), e127-e131. doi: 10.1111/j.15281167.2012.03509 .x

Kothare, S. V., VanLandingham, K., Armon, C., Luther, J. S., Friedman, A., \& Radtke, R. A. (1998). Seizure onset from periventricular nodular heterotopias: Depth-electrode study. Neurology, 51(6), 1723-1727. doi: 10.1212 /WNL. 51.6.1723

Lambrecq V., Lehongre K., Adam C, Frazzini V., Mathon B., Clemenceau S., Hasboun D., Charpier S., Baulac M., Navarro V., Le Van Quyen M. (2017). Single-unit activities during the transition to seizures in deep mesial structures. Ann Neurol., Dec; 82(6):1022-1028. doi: 10.1002/ana.25111.

Le Van Quyen, M., Bragin, A., Staba, R., Crepon, B., Wilson, C. L., \& Engel, J. (2008). Cell type-specific firing during ripple oscillations in the hippocampal formation of humans. The Journal of Neuroscience: The Official Journal of the Society for Neuroscience, 28(24), 61046110. doi: 10.1523 /JNEUROSCI. 0437-08.2008

Maris, E., \& Oostenveld, R. (2007). Nonparametric statistical testing of EEG- and MEG-data. Journal of Neuroscience Methods, 164(1), 177-190. doi: 10.1016 /j.jneumeth. 2007.03.024 
Meroni, A., Galli, C., Bramerio, M., Tassi, L., Colombo, N., Cossu, M., ... Spreafico, R. (2009). Nodular heterotopia: A neuropathological study of 24 patients undergoing surgery for drugresistant epilepsy. Epilepsia, 50(1), 116-124. doi: 10.1111/j.15281167.2008.01717 .x

Mirandola, L., Mai, R. F., Francione, S., Pelliccia, V., Gozzo, F., Sartori, I., ... Tassi, L. (2017).

Stereo-EEG: Diagnostic and therapeutic tool for periventricular nodular heterotopia epilepsies. Epilepsia, 58(11), 1962-1971. doi: 10.1111/epi. 13895

Najm I.M., Tilelli C.Q., Oghlakian R. (2007) Pathophysiological mechanisms of focal cortical dysplasia: a critical review of human tissue studies and animal models. Epilepsia; 48 Suppl 2:2132.

Oostenveld, R., Fries, P., Maris, E., \& Schoffelen, J.M. (2011). FieldTrip: Open Source Software for Advanced Analysis of MEG, EEG, and Invasive Electrophysiological Data. Computational Intelligence and Neuroscience, 2011, 1-9. doi: 10.1155/2011/156869

Palmini, A., Gambardella, A., Andermann, F., Dubeau, F., da Costa, J. C., Olivier, A., ... Kim, H.I. (1995, April). Intrinsic epileptogenicity of human dysplastic cortex as suggested by corticography and surgical results. Annals of Neurology, 37(4), 476-487. doi: 10.1002 /ana. 410370410

Pérez-García F., Lehongre K., Bardinet E., Jannin P., Navarro V., Hasboun D., Fernandez-Vidal S. 2015. Automatic segmentation of depth electrodes implanted in epileptic patients: a modular tool adaptable to multicentric protocols. 31st International Epilepsy Congress. (https://icminstitute.org/fr/cenir-stim-stereotaxy-core-facility-techniques-images-models/) 
Petit LF., Jalabert M., Buhler E., Malvache A., Peret A., Chauvin Y., Watrin F., Represa A., Manent JB. (2014). Normotopic cortex is the major contributor to epilepsy in experimental double cortex. Ann Neurol. Sep;76(3):428-42. doi: 10.1002/ana.24237.

Pizzo F., Roehri N., Catenoix H., Medina S., McGonigal A., Giusiano B., Carron R., Scavarda D., Ostrowsky K., Lepine A., et al. (2017). Epileptogenic networks in nodular heterotopia: A stereoelectroencephalography study. Epilepsia. Dec; 58(12):2112-2123. doi: 10.1111/epi.13919.

Shinomoto, S., Shima, K., \& Tanji, J. (2003). Differences in spiking patterns among cortical neurons. Neural Computation, 15(12), 2823-2842. doi: 10.1162/089976603322518759

Tassi, L. (2004). Electroclinical, MRI and neuropathological study of 10 patients with nodular heterotopia, with surgical outcomes. Brain, 128(2), 321-337. doi: 10.1093/brain/awh 357

Tóth K., Hofer K.T., Kandrács Á., Entz L., Bagó A.G., Erőss L., Jordán Z., Nagy G., Fabó D., Ulbert I., Wittner L. (2018). Hyperexcitability of the network contributes to synchronization processes in the human epileptic neocortex. J Physiol 15; 596(2):317-342

Thom, M., Martinian, L., Parnavelas, J. G., \& Sisodiya, S. M. (2004, August). Distribution of Cortical Interneurons in Grey Matter Heterotopia in Patients with Epilepsy. Epilepsia, 45(8), 916-923. doi: 10.1111/j.00139580.2004.46603.

Valton, L., Guye, M., McGonigal, A., Marquis, P., Wendling, F., R’egis, J., Bartolomei, F., et al. (2008). Functional interactions in brain networks underlying epileptic seizures in bilateral diffuse periventricular heterotopia. Clinical Neurophysiology, 119(1), 212-223. 
Yger, P., Spampinato, G. L., Esposito, E., Lefebvre, B., Deny, S., Gardella, C., Marre, O. et al. (2018). A spike sorting toolbox for up to thousands of electrodes validated with ground truth recordings in vitro and in vivo. eLife, 7. doi: 10.7554 /eLife. 34518 


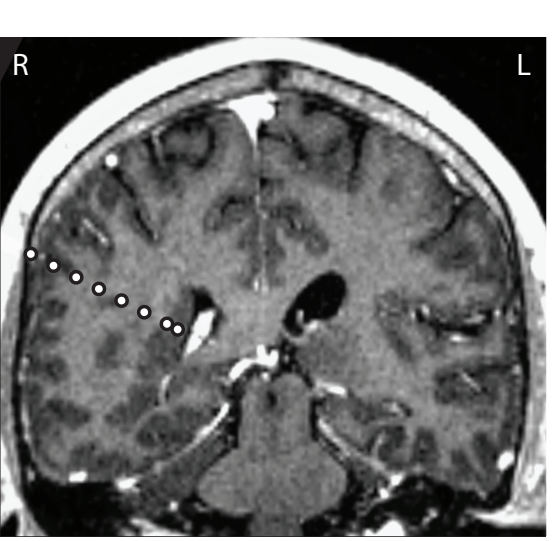

D Implantation Nodule 2 (Patient 2)

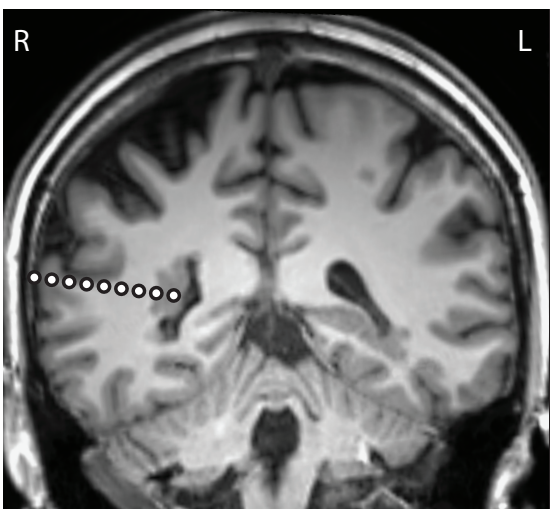

G Implantation Nodule 3 (Patient 2)

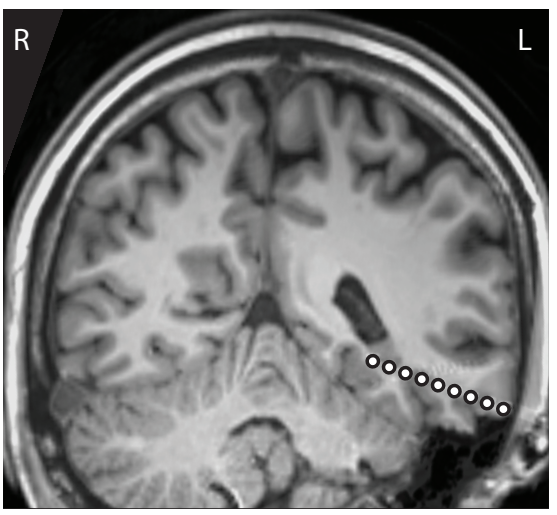

J Seizure onset Nodule 1 (Patient 1)

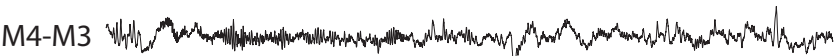
M3-M4 M2-M3 M1-M2

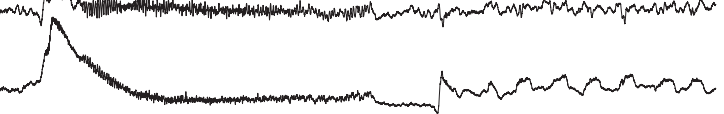

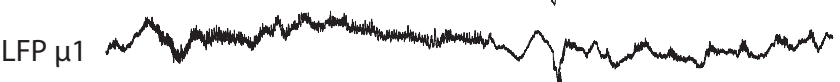
LFP H2

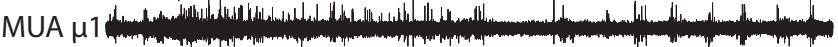
MUA $\mu 2$

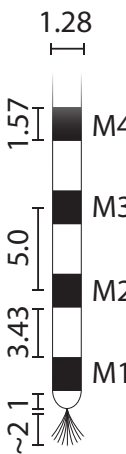

$\mu 1-\mu 8$
M2-M3

Epileptic Spike

Fast Activity

Periodic Slow Waves

M1-M2

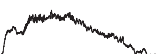

2.50

Time (s)

2.5

hamann -200

Mumandand $M$ Mn

$-50$

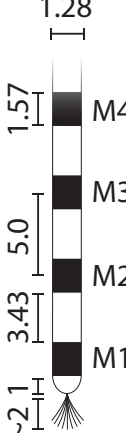

$\mu 1-\mu 8$
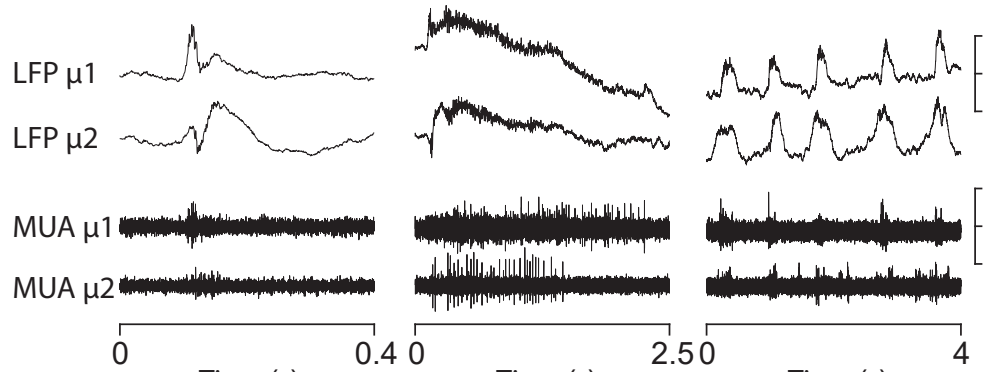

Time (s)

Time (s)

Epileptic Spike

Periodic Fast Activity

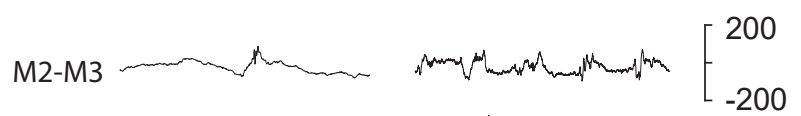

M1-M2

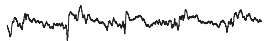

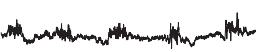

$-50$

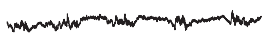

50

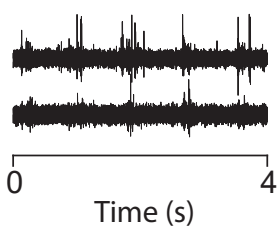

$-50$

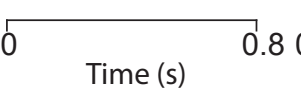

200

$-200$

0

50 
A Waveform

ISI
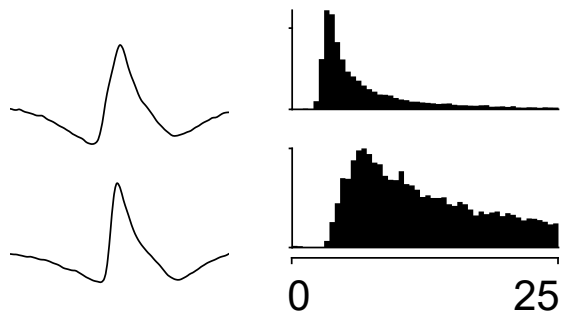

B Waveform
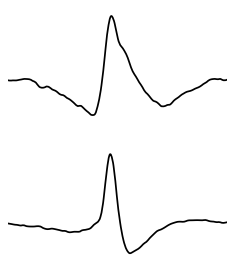

C Waveform
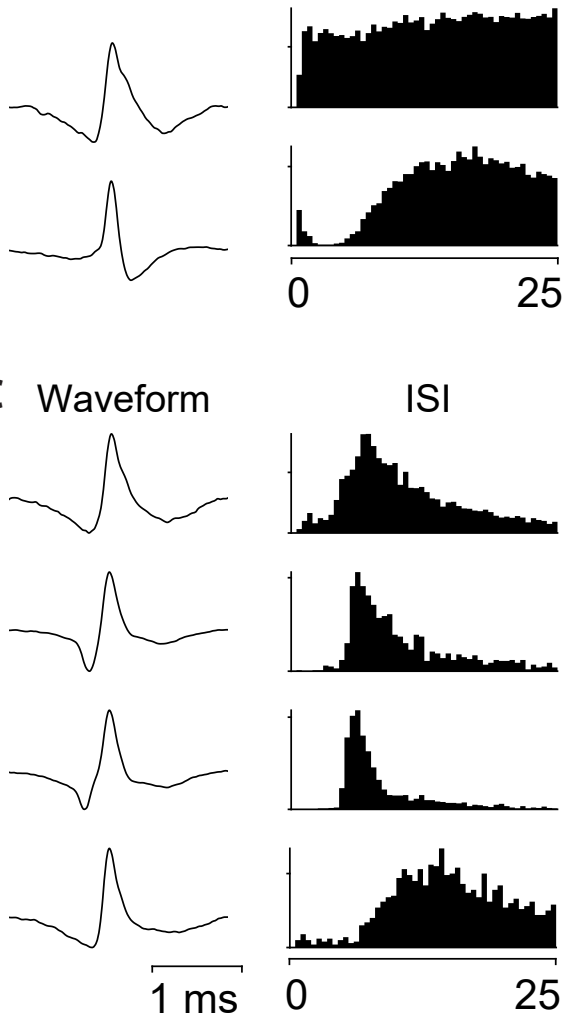

Epileptic Spike

Fast Activity

Period Slow Wave
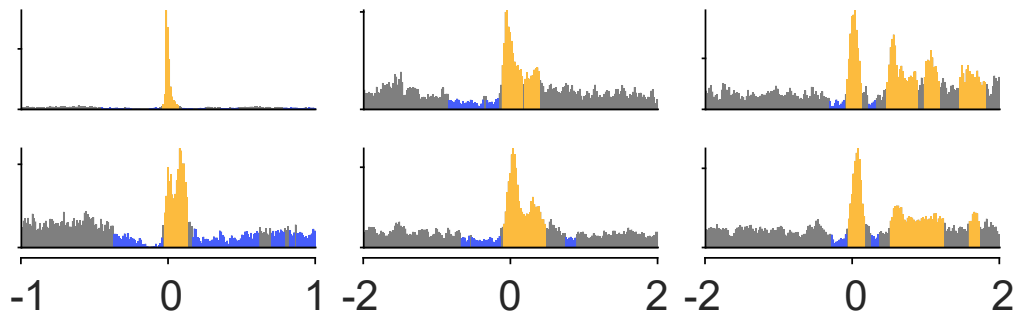

Epileptic Spike

Fast Activity

Period Slow Wave
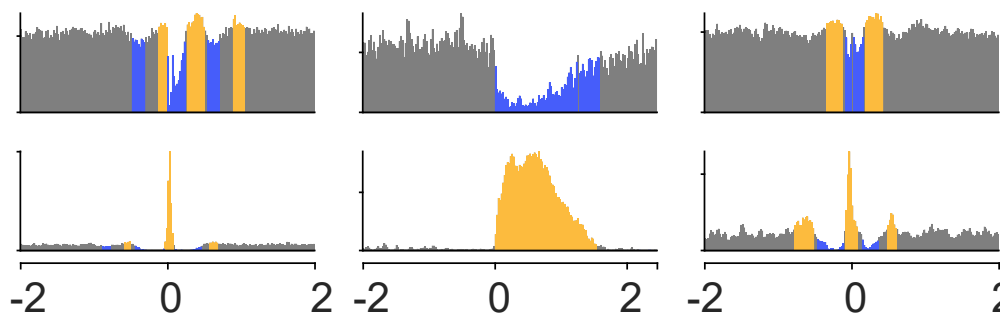

Epileptic Spike Periodic Fast Activity
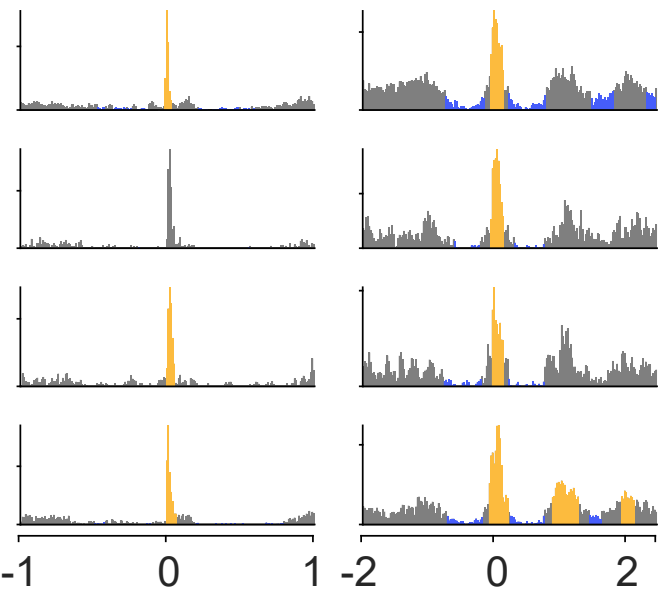

D

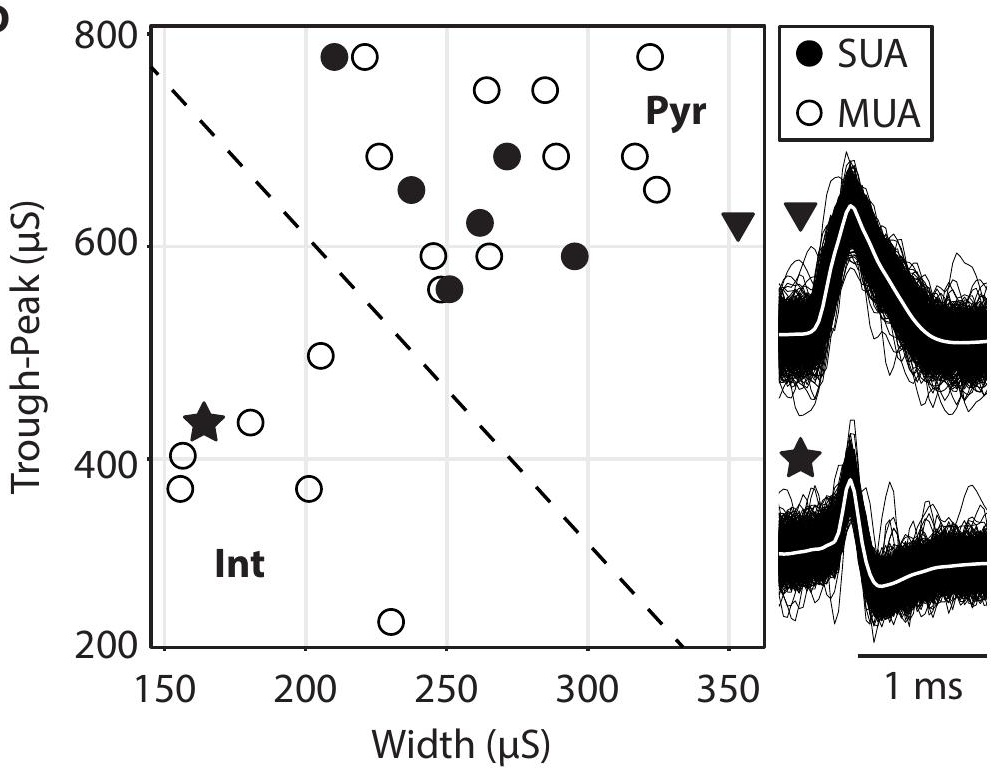


Epileptic Spike

Fast Activity Periodic Slow Wave

\begin{tabular}{|llll|lll|lll|lll|l|}
\hline $\mathrm{N}$ & ID & $\#$ & RPV & $\uparrow$ & $\downarrow$ & $\rho$ & $\uparrow$ & $\downarrow$ & $\rho$ & $\uparrow$ & $\downarrow$ & $\rho$ & F \\
\hline 1 & 2 & 35402 & 0.09 & $3840 \ddagger$ & $95 \ddagger$ & $0.68 \ddagger$ & $406 \ddagger$ & $83 \ddagger$ & $0.63 \ddagger$ & $355 \ddagger$ & $82 \ddagger$ & $0.79 \ddagger$ & $\mathrm{A}$ \\
& 7 & 35603 & 0.03 & $285 \ddagger$ & $98 \ddagger$ & $0.50 \ddagger$ & $493 \ddagger$ & $69 \ddagger$ & $0.68 \ddagger$ & $128 \ddagger$ & $76 \ddagger$ & $0.70 \ddagger$ & \\
& 1 & 324414 & 3.32 & $373 \ddagger$ & $69 \ddagger$ & $0.74 \ddagger$ & $319 \ddagger$ & $48 \ddagger$ & $0.54 \ddagger$ & $153 \ddagger$ & $54 \ddagger$ & $0.84 \ddagger$ & \\
& 3 & 59035 & 0.77 & $2421 \ddagger$ & $84 \ddagger$ & $0.66 \ddagger$ & $238 \ddagger$ & $58 \ddagger$ & $0.60 \ddagger$ & $180 \ddagger$ & $71 \ddagger$ & $0.82 \ddagger$ & \\
& 4 & 9032 & 0.43 & $3074 \ddagger$ & $100 \ddagger$ & $0.75 \ddagger$ & $361 \ddagger$ & $96 \ddagger$ & $0.47 \ddagger$ & $201 \ddagger$ & $81 \ddagger$ & $0.80 \ddagger$ & \\
& 5 & 75177 & 1.48 & $1575 \ddagger$ & $65 \ddagger$ & $0.90 \ddagger$ & $249 \ddagger$ & $63 \ddagger$ & $0.54 \ddagger$ & $305 \ddagger$ & $61 \ddagger$ & $0.88 \ddagger$ & \\
& 6 & 28386 & 0.72 & $1144 \ddagger$ & $97 \ddagger$ & $0.72 \ddagger$ & $468 \ddagger$ & $79 \ddagger$ & $0.69 \ddagger$ & $216 \ddagger$ & $66 \ddagger$ & $0.66 \ddagger$ & \\
\hline
\end{tabular}

Epileptic Spike

Fast Activity

Periodic Slow Wave

\begin{tabular}{|llll|lll|lll|lll|l|}
\hline $\mathrm{N}$ & ID & $\#$ & RPV & $\uparrow$ & $\downarrow$ & $\rho$ & $\uparrow$ & $\downarrow$ & $\rho$ & $\uparrow$ & $\downarrow$ & $\rho$ & $\mathrm{F}$ \\
\hline 2 & 5 & 177686 & 0.90 & $24 \ddagger$ & $91 \ddagger$ & $-0.45 \ddagger$ & n.s. & $93 \ddagger$ & $-0.68 \ddagger$ & $22 \ddagger$ & $26 \ddagger$ & $-0.68 \ddagger$ & $\mathrm{C}$ \\
& 8 & 22797 & 0.67 & $1582 \ddagger$ & $97 \ddagger$ & $0.67 \ddagger$ & 4281 & $100 *$ & $0.86 \ddagger$ & $505 \ddagger$ & $98 \ddagger$ & $0.83 \ddagger$ & \\
& 1 & 6523 & 0.14 & $524 \ddagger$ & $100 \ddagger$ & $0.44 \ddagger$ & n.s. & n.s. & $0.07 \ddagger$ & $170 \ddagger$ & n.s. & $0.43 \ddagger$ & \\
& 2 & 11431 & 0.45 & $599 \ddagger$ & $96 \ddagger$ & $0.45 \ddagger$ & n.s. & $100 \ddagger$ & $0.09 \ddagger$ & $105 \ddagger$ & $60 *$ & $0.39 \ddagger$ & \\
& 3 & 92917 & 1.78 & $1058 \ddagger$ & $99 \ddagger$ & $0.59 \ddagger$ & $646 \ddagger$ & $100 \ddagger$ & $0.53 \ddagger$ & $392 \ddagger$ & $91 \ddagger$ & $0.56 \ddagger$ & \\
& 4 & 20365 & 0.56 & n.s. & $60 \ddagger$ & $-0.16 \ddagger$ & n.s. & $100 \ddagger$ & $-0.29 \ddagger$ & n.s. & n.s. & $-0.26 \ddagger$ & \\
& 6 & 56998 & 0.57 & $40 \ddagger$ & $52 \ddagger$ & $0.28 \ddagger$ & n.s. & n.s. & n.s. & n.s. & n.s & $0.24 \ddagger$ & \\
& 7 & 15479 & 2.47 & $900 \ddagger$ & $89 \ddagger$ & $0.71 \ddagger$ & 1536 & $100 \ddagger$ & $0.85 \ddagger$ & $231 \ddagger$ & $60 \ddagger$ & $0.75 \ddagger$ & B \\
& 9 & 79082 & 3.36 & $744 \ddagger$ & $97 \ddagger$ & $0.80 \ddagger$ & 1484 & $100 \ddagger$ & $0.69 \ddagger$ & $264 \ddagger$ & $87 \ddagger$ & $0.74 \ddagger$ & \\
& 10 & 156754 & 2.52 & $153 \ddagger$ & $78 \ddagger$ & $0.64 \ddagger$ & $118 \ddagger$ & $73 \ddagger$ & $0.49 \ddagger$ & $35 \ddagger$ & $39 \ddagger$ & $0.44 \ddagger$ & \\
\hline
\end{tabular}

Epileptic Spike

Periodic Fast Activity

\begin{tabular}{|llll|lll|lll|l|l|}
\hline $\mathrm{N}$ & ID & $\#$ & RPV & $\uparrow$ & $\downarrow$ & $\rho$ & $\uparrow$ & $\downarrow$ & $\rho$ & & F \\
\hline 3 & 2 & 24774 & 0.48 & $1905 \ddagger$ & $100 \ddagger$ & $-0.12 \ddagger$ & $297 \ddagger$ & $98 \ddagger$ & $0.70 \ddagger$ & & \\
& 4 & 5020 & 0.02 & n.s. & $100 \ddagger$ & $0.39 \ddagger$ & $588 *$ & $100 \ddagger$ & $0.65 \ddagger$ & & \\
& 5 & 4429 & 0.00 & $1968 *$ & $100 \ddagger$ & $0.49 \ddagger$ & $467 *$ & $100 \ddagger$ & $0.66 \ddagger$ & & \\
& 7 & 13844 & 0.14 & $1825 *$ & $100 \ddagger$ & $0.05 *$ & $543 \ddagger$ & $100 \ddagger$ & $0.67 \ddagger$ & & \\
& 1 & 103242 & 3.87 & $1796 \ddagger$ & $96 \ddagger$ & $0.36 \ddagger$ & $181 \ddagger$ & $91 \ddagger$ & $0.74 \ddagger$ & & \\
& 3 & 43938 & 1.33 & $1153 \ddagger$ & $99 \ddagger$ & $0.37 \ddagger$ & $291 \ddagger$ & $93 \ddagger$ & $0.50 \ddagger$ & & \\
& 6 & 23329 & 0.90 & $1042 \ddagger$ & $94 \ddagger$ & $0.26 \ddagger$ & $267 \ddagger$ & $78 \ddagger$ & $0.78 \ddagger$ & & \\
& 8 & 11527 & 0.65 & $1232 *$ & $100 \ddagger$ & $0.05^{*}$ & $465 \ddagger$ & $100 \ddagger$ & $0.75 \ddagger$ & & \\
\hline
\end{tabular}

Table 1. Spike analysis summary. $\mathrm{N}=$ Nodule, $\mathrm{ID}=$ unit, $\#=$ number of action potentials.

Increases $(\uparrow)$ and decreases $(\downarrow)$ in firing-rate of $\%$ baseline, for most significant temporal cluster $($ n.s. $=$ non-significant $), \rho=$ Pearson correlation between spike-rate and LFP. F2 = Panel of Figure 2 for more detailed presentation of cell behavior. Single units are highlighted in gray (see also Supplemental Figure 3). $*=p<0.025, \dagger=p<0.01, \ddagger=p<0.001$. 